\title{
INTERACTION OF PLANT ALKALOID, BERBERINE, WITH ZWITTERIONIC AND NEGATIVELY CHARGED PHOSPHOLIPID BILAYERS
}

\author{
JUSTYNA GĄSIOROWSKA, OLGA WESOLOWSKA, KRYSTYNA MICHALAK \\ Department of Biophysics, Wrocław Medical University, Wrocław, Poland
}

Received April 28, 2011; accepted October 05, 2011; published online October 07, 2011

\begin{abstract}
Berberine exhibits many pharmacological activities e.g. antibacterial, anti-inflammatory, antiproliferative and apoptosis-inducing. Interaction of berberine with model membranes was studied for the first time using differential scanning calorimetry, fluorescence spectroscopy and turbidity measurements. Influence of berberine on thermotropic properties of bilayers formed from zwitterionic DMPC was insignificant, whereas in bilayers formed from negatively charged DMPG berberine reduced the temperature and cooperativity of main phospholipid phase transition. In higher concentrations berberine induced complex double-peak transition, with the new peak appearing in temperature higher than the original one. It suggested the interaction of the alkaloid with lipid headgroup region of the bilayer. Additionally, berberine quenched fluorescence of Prodan to a higher extent than Laurdan that pointed to stronger interaction with membrane segments close to its surface. Berberine-induced fluorescence quenching of both probes was more pronounced in DPPG than in DPPC bilayers. It was concluded that electrostatic interactions governed berberine association with model membrane.
\end{abstract}

\section{INTRODUCTION}

Berberine (Fig. 1) is an isoquinoline alkaloid present in various plant species that have been used in traditional medicine throughout the world. These include European barberry (Berberis vulgaris), Indian barberry (Berberis aristata), Oregon-grape (Mahonia aquifolium), goldenseal (Hydrastis canadensis), yellow-fruit moonseed (Arcangelisia flava), and Chinese goldthread (Coptis chinensis) (Imanshahidi \& Hosseinzadeh, 2008). The list of traditional uses of berberine-containing plants is very long (see (Imanshahidi \& Hosseinzadeh, 2008) for a review) since berberine exhibits a variety of biological and pharmacological effects. It possesses antiinflammatory (Ivanovska \& Philipov, 1996; Kuo et al., 2004), antibacterial (Scazzocchio et al., 2001; Freile et al., 2003) and anti-fungal activity (Iwazaki et al., 2010). Several studies have indicated that in some types of cancer cells berberine is a potent antiproliferative, apoptosis-inducing and cell cycle-arresting agent (Jantova et al., 2007; Gupta et al., 2010; Wang et al., 2010). Anti-tumor activity of berberine has been partially attributed to its ability to induce double strand breaks in DNA (Liu et al., 2009). Additionally, berberine was reported to bind to poly(A) sequences in RNA (Giri \& Kumar, 2010). Moreover, the alkaloid has been shown to exert immunomodulatory and cardiovascular effects (Imanshahidi \& Hosseinzadeh,
2008). Recently, berberine has also attracted attention as a potential therapeutic for nervous system disorders (for a review see (Howes \& Houghton, 2003; Kulkarni \& Dhir, 2010)).<smiles>COc1ccc2cc3c4cc5c(cc4cc[n+]3cc2c1OC)OCO5</smiles>

Fig. 1. Chemical structure of berberine.

In spite of wide interest in pharmacological activities of berberine, its influence on biological membranes remains a non-studied area. Rackova et al. (2004) showed that berberine did not possess any antioxidant activity in DOPC liposomal membranes. The aim of the present study was to investigate the interaction of berberine with model membranes formed from zwitterionic phosphatidylcholine as well as from negatively charged phosphatidylglycerol by means of 
differential scanning calorimetry (DSC), fluorescence spectroscopy, and turbidimetry. It was concluded that the electrostatic interaction between positively charged nitrogen of berberine and negatively charged lipid headgroups governed alkaloid association with model membrane. According to our best knowledge, this report constitutes the first study on the interaction of berberine with phospholipid bilayer.

\section{MATERIALS AND METHODS}

DMPC (1,2-dimyristoyl-sn-glycero-3-phosphocholine) and DMPG (1,2-dimyristoyl-sn-glycero-3- phosphoglycerol) were purchased from Sigma (Poznań, Poland). DPPC (1,2-dipalmitoyl-sn-glycero-3-phosphocholine) and DPPG (1,2-dipalmitoyl-sn-glycero-3-phosphoglycerol) were from Avanti Polar Lipids (Alabaster, AL, USA). Laurdan (2-(dimethylamino)-6-dodecanoylnaphthalene) and Prodan (2-(dimethylamino)-6propionylnaphthalene) were from Molecular Probes (Eugenie, OR, USA). Laurdan and Prodan were dissolved in DMSO to obtain $1 \mathrm{mM}$ stock solutions.

Berberine was purchased from Alexis Biochemicals (Lausen, Switzerland). For microcalorimetric measurements, berberine was dissolved in methanol to obtain $3.5 \mathrm{mM}$ stock solution. To prepare berberine stock solution $(30 \mathrm{mM})$ for fluorescence spectroscopy measurements, berberine was dissolved in methanol:water mixture $(2: 1)$.

\section{Differential scanning calorimetry}

To prepare the sample, $0.002 \mathrm{~g}$ of DMPC or DMPG was mixed with methanol solution of berberine to obtain the desired molar ratio. Next, the lipid mixture was evaporated under stream of nitrogen and placed under vacuum for $2 \mathrm{~h}$. The dry samples were hydrated by adding $15 \mu \mathrm{l}$ of $20 \mathrm{mM}$ Tris- $\mathrm{HCl}$ buffer $(150 \mathrm{mM} \mathrm{NaCl}$, $0.5 \mathrm{mM}$ EDTA, pH 7.4). The hydrated mixtures were heated to $50{ }^{\circ} \mathrm{C}$ and vortexed until homogeneous dispersion was obtained. Samples were then sealed in aluminum pans and scanned at a rate of $1{ }^{\circ} \mathrm{C} / \mathrm{min}$ immediately after preparation. Heating scans were recorded. Calorimetric measurements were performed using a DSC 600 microcalorimeter (Unipan, Warsaw, Poland). Samples were prepared in duplicate, and each sample was scanned at least four times. The calorimetric data was collected and processed off-line using software developed in our laboratory.

\section{Fluorescence spectroscopy}

As model membranes, small unilamellar liposomes formed from DPPC and DPPG were used. Liposomes were obtained by sonication of lipid suspensions in 20 $\mathrm{mM}$ Tris- $\mathrm{HCl}$ buffer $(150 \mathrm{mM} \mathrm{NaCl}, 0.5 \mathrm{mM}$ EDTA, $\mathrm{pH}$ 7.4) using UP 200s sonicator (Dr Hilscher, Berlin,
Germany). Samples were sonicated until the liposome suspension became opalescent. Liposomes $(200 \mu \mathrm{M}$ of lipid) were incubated with $5 \mu \mathrm{M}$ of fluorescent probe (Laurdan or Prodan) for $30 \mathrm{~min}$ (room temperature, darkness). Then the berberine solutions were added and the samples were incubated for a further $20 \mathrm{~min}$ (darkness, room temperature). For each berberine concentration a separate sample was prepared. The fluorescence emission spectra were collected using a LS 50B spectrofluorimeter (Perkin-Elmer Ltd., Beaconsfiled, UK) equipped with a xenon lamp. The excitation wavelengths were $380 \mathrm{~nm}$ for Prodan and 390 $\mathrm{nm}$ for Laurdan. It was checked that berberine itself did not exhibit fluorescence in this region. Both the excitation and emission slit widths were $5 \mathrm{~nm}$. All experiments were performed at $25^{\circ} \mathrm{C}$. The content of a cuvette was continuously stirred with a magnetic stirrer. For monitoring phospholipid phase transition with the use of Laurdan the parameter of generalized polarization (GP) was used, defined as in Parasassi et al. (1990):

$$
G P=\frac{\left(I_{B}-I_{R}\right)}{\left(I_{B}+I_{R}\right)}
$$

where $I_{B}$ and $I_{R}$ are the fluorescence emission intensities at the blue and the red edge of the emission spectrum, respectively. $I_{B}$ was measured at $440 \mathrm{~nm}$, while $I_{R}$ at 490 $\mathrm{nm}$. Experiments were performed in triplicate.

\section{Turbidity measurements}

Unilamellar DPPC and DPPG liposomes were prepared to investigate berberine-induced turbidity changes in suspension by absorbance measurement at $500 \mathrm{~nm}$. The absorbance of liposome dispersions was used as a qualitative measure of liposome aggregation. The lipids were dissolved in chloroform:methanol (1:1), and the solvent was evaporated under a stream of nitrogen and placed under vacuum for $2 \mathrm{~h}$. In the following step, lipids were hydrated using $20 \mathrm{mM}$ Tris buffer (0.1 mM EDTA, pH 7.4). The unilamellar liposomes were obtained by seven-fold extrusion through polycarbonate filter with pore size $200 \mu \mathrm{m}$. For each sample absorbance was measured for $500 \mathrm{~s}$ at $1 \mathrm{~s}$ intervals after addition of berberine solution to liposome suspension. The berberine:lipid molar ratio in liposome dispersions was 2.5 , the final lipid concentration was 50 $\mu \mathrm{M}$. Measurements were performed using Lambda Bio 20 (Perkin-Elmer, USA) spectrophotometer. In control experiments it was checked that the solvent used to dissolve berberine did not cause liposome aggregation. The absorbance was measured at $500 \mathrm{~nm}$ as no absorbance of berberine was recorded at this wavelength. 
RESULTS

Differential scanning calorimetry

To estimate the role of lipid charge in the interaction of plant alkaloid berberine with membranes its effect on zwitterionic phospholipid DMPC and acidic DMPG was studied. The thermograms of DMPC in the absence of berberine and in increasing contents of the studied compound are presented in Fig. 2 and the parameters describing main phospholipid phase transition can be found in Tab. 1. In pure DMPC two phase transitions were observed - a pretransition and a gel-liquid crystalline transition. At berberine:DMPC molar ratio 0.06 and 0.12 the pretransition was still visible in the thermograms, it disappeared completely only in higher alkaloid concentrations. The addition of berberine to DMPC model membranes did not change the temperature of phospholipid main phase transition significantly, even if compound:lipid molar ratio was as high as 0.24 . Also half-height widths of calorimetric peaks were not significantly altered in the presence of the alkaloid. On the other hand, the transition enthalpy was affected by berberine. At berberine:DMPC molar ratio of 0.24 this parameter was reduced almost four times in comparison with the pure lipid. It was concluded that high compound:lipid molar ratios had to be applied to observe significant changes induced by berberine in zwitterionic DMPC bilayers.

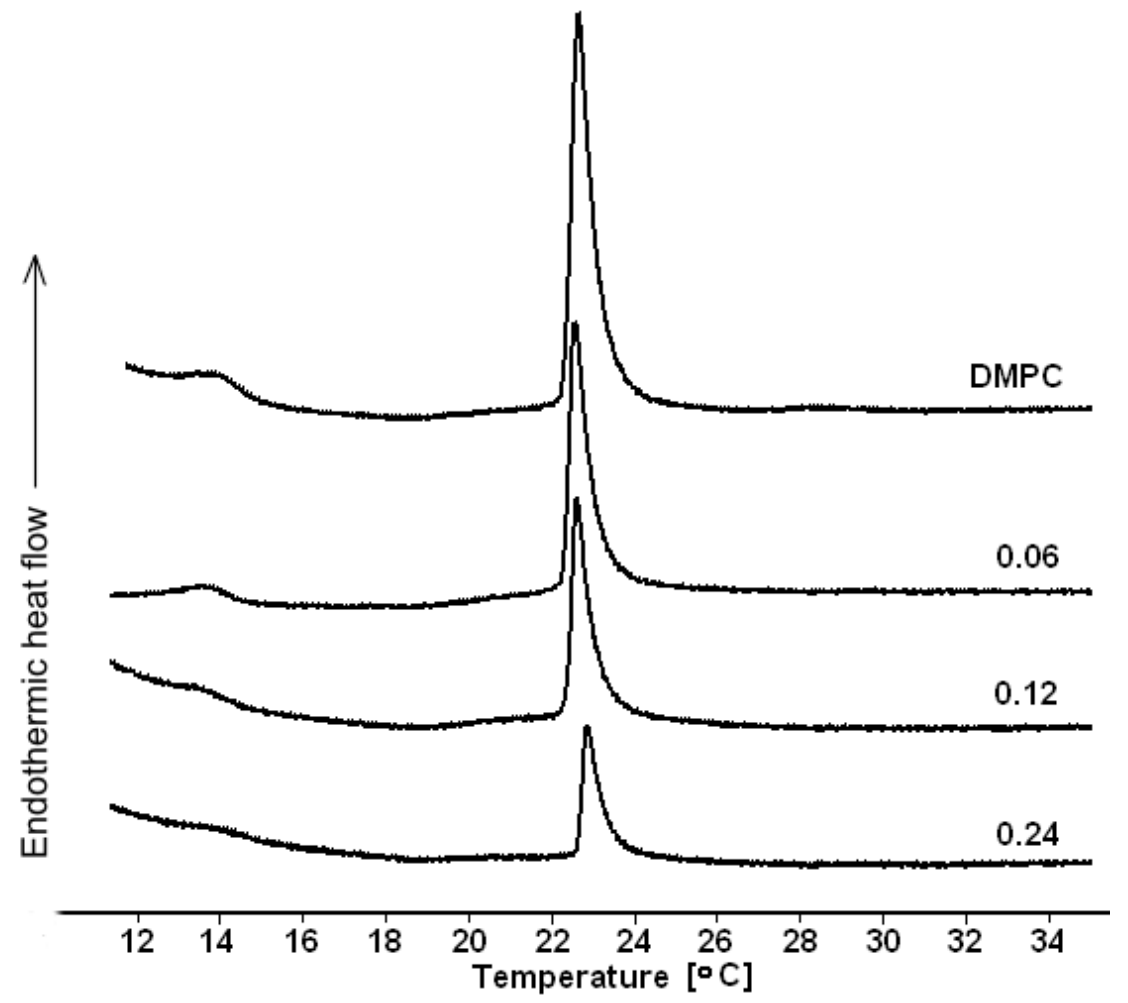

Fig. 2. Thermograms of DMPC and berberine-DMPC mixtures. Numbers in the figure represent berberine:DMPC molar ratios. The thermograms were normalized to an equal amount of lipid for each profile.

Table 1. Main phospholipid phase transition parameters of DMPC and berberine-DMPC mixtures. Values are presented as means \pm S.D.

\begin{tabular}{cccc}
\hline $\begin{array}{c}\text { Berberine:DMPC molar } \\
\text { ratio }\end{array}$ & $\begin{array}{c}\text { Main phase transition } \\
\text { temperature }\left[{ }^{\circ} \mathrm{C}\right]\end{array}$ & $\begin{array}{c}\text { Transition enthalpy } \\
{[\mathrm{kJ} / \mathrm{mol}]}\end{array}$ & Half-height width [ $\left.{ }^{\circ} \mathrm{C}\right]$ \\
0 & $23.00 \pm 0.16$ & $26.00 \pm 0.00$ & $0.50 \pm 0.06$ \\
0.06 & $22.86 \pm 0.21$ & $14.27 \pm 2.45$ & $0.45 \pm 0.04$ \\
0.12 & $22.87 \pm 0.16$ & $15.78 \pm 3.76$ & $0.46 \pm 0.04$ \\
0.24 & $22.84 \pm 0.08$ & $6.93 \pm 2.30$ & $0.43 \pm 0.01$ \\
\hline
\end{tabular}


In model membranes composed of acidic DMPG both pretransition and gel-liquid crystalline transition were observed (see Fig. 3 for the thermograms). Pretransition disappeared at the lowest berberine:DMPG molar ratio studied (0.02). The calorimetric peaks mirroring the main phospholipid phase transition were broadened in the presence of the alkaloid, and the transition temperature was decreased (Tab. 2). Starting from berberine:DMPG molar ratio 0.04 transition peaks became asymmetric with high-temperature shoulders broader than the low-temperature ones. Above this molar ratio berberine induced a complex double-peak transition. The presence of berberine did not affect transition enthalpy significantly with the exception of compound:lipid molar ratio of 0.08 at which the enthalpy was reduced by c.a. $20 \%$. It can be seen that much lower concentrations of berberine were needed to modify the parameters of DMPG phase transition as compared with DMPC.

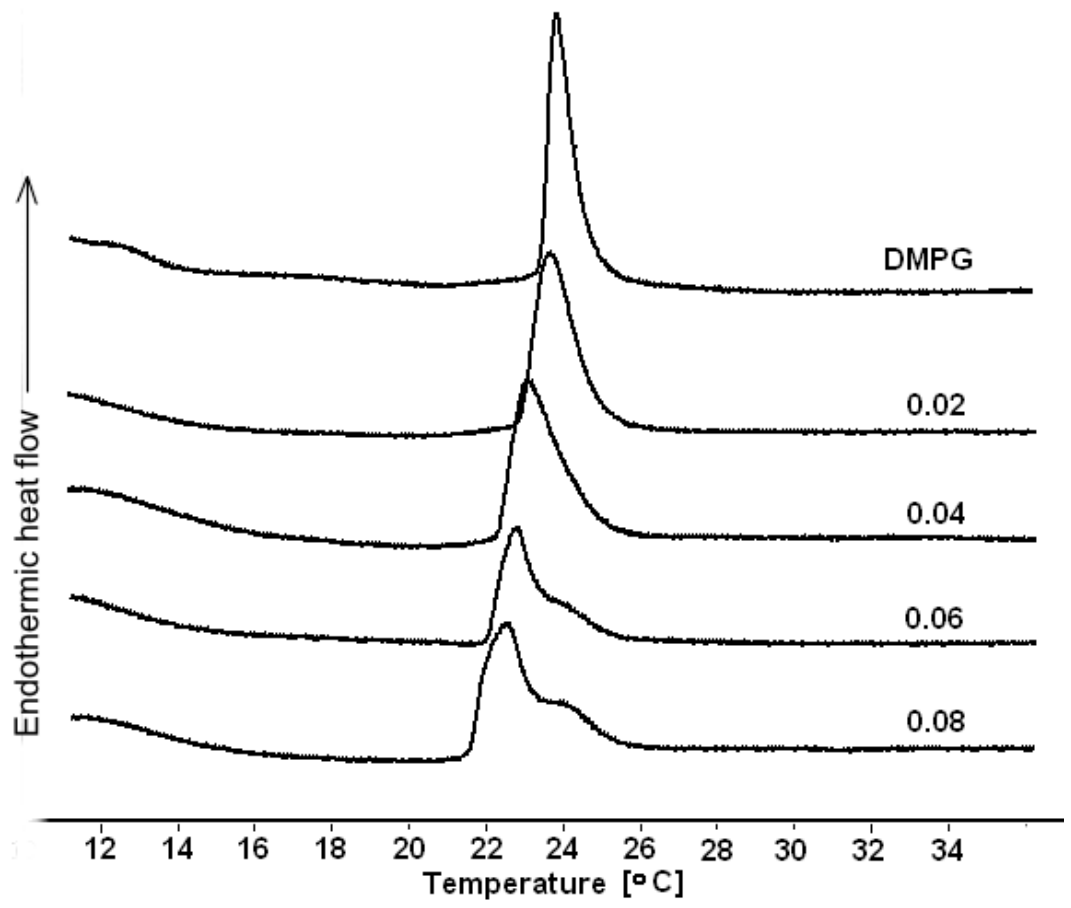

Fig. 3. Thermograms of DMPG and berberine-DMPG mixtures. Numbers in the figure represent berberine:DMPG molar ratios. The thermograms were normalized to an equal amount of lipid for each profile.

Table 2. Main phospholipid phase transition parameters of DMPG and berberine-DMPG mixtures. Values are presented as means \pm S.D.

\begin{tabular}{cccc}
\hline $\begin{array}{c}\text { Berberine:DMPG molar } \\
\text { ratio }\end{array}$ & $\begin{array}{c}\text { Main phase transition } \\
\text { temperature }\left[{ }^{\circ} \mathrm{C}\right]\end{array}$ & $\begin{array}{c}\text { Transition enthalpy } \\
{[\mathrm{kJ} / \mathrm{mol}]}\end{array}$ & Half-height width [ $\left.{ }^{\circ} \mathrm{C}\right]$ \\
0 & $24.00 \pm 0.10$ & $28.50 \pm 0.00$ & $0.71 \pm 0.05$ \\
0.02 & $23.76 \pm 0.26$ & $30.87 \pm 3.13$ & $1.05 \pm 0.14$ \\
0.04 & $23.69 \pm 0.11$ & $29.83 \pm 1.20$ & $1.19 \pm 0.10$ \\
0.06 & $23.19 \pm 0.24$ & $27.67 \pm 3.43$ & $1.09 \pm 0.08$ \\
0.08 & $23.02 \pm 0.17$ & $22.18 \pm 0.85$ & $1.57 \pm 0.54$ \\
\hline
\end{tabular}

\section{Fluorescence spectroscopy}

To further study the effect of berberine on PC and PG bilayers we have used two fluorescent probes localized in different membrane segments. Laurdan and Prodan both possess the same fluorophore connected to an alkyl chain of different length (three carbon atoms in Prodan and twelve in Laurdan). Therefore Prodan molecules locate closer to the hydrophilic surface of a bilayer than
Laurdan (Krasnowska et al., 1998) whose fluorophore is positioned close to phospholipid glycerol groups (Bagatolli et al., 1999). In both lipid systems, DPPC and DPPG, quenching of Prodan (Fig. 4, circles) was significantly stronger than quenching of Laurdan (Fig. 4, squares). Furthermore, quenching of both fluorescent probes in liposomes formed from zwitterionic DPPC 
was weaker than in negatively charged DPPG liposomes.

Additionally, the influence of berberine at $25 \mu \mathrm{M}$ concentration on main DPPC and DPPG phase transition was monitored by the use of Laurdan generalized polarization and no influence of the studied alkaloid on any of the lipids was observed (data not shown). The relatively low concentration of berberine was chosen for this experiment as this was the highest concentration at which Laurdan fluorescence spectra intensity was high enough to estimate GP values reliably.

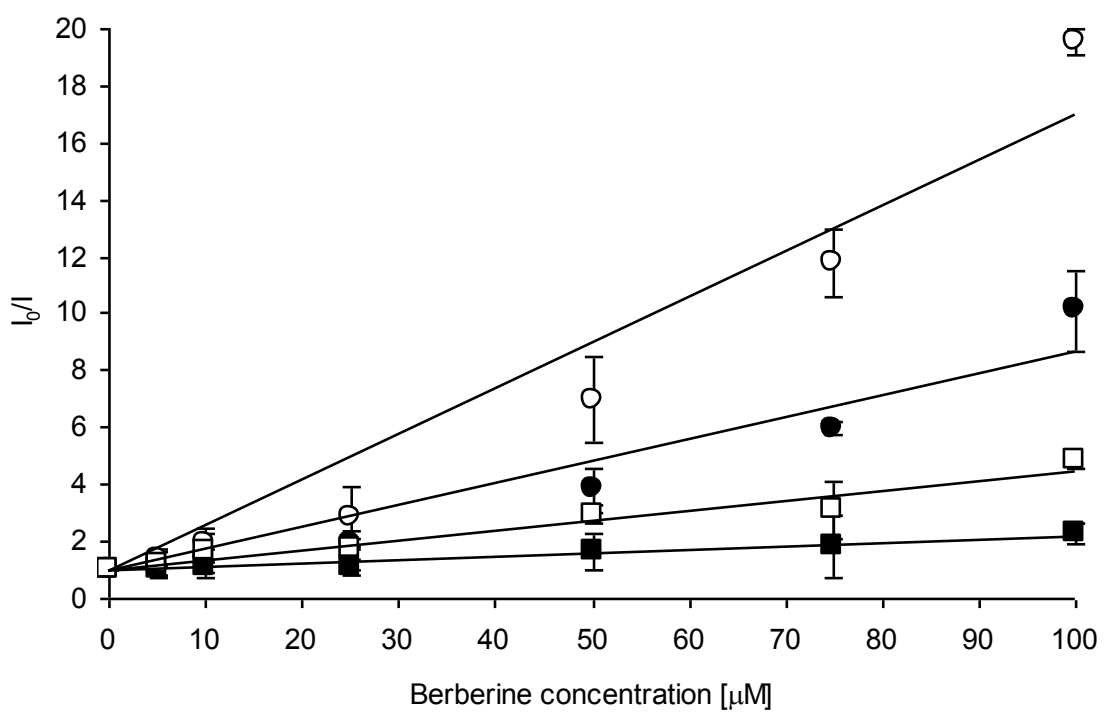

Fig. 4. Stern-Volmer plots for quenching of Prodan (circles) and Laurdan (squares) fluorescence by berberine in DPPC (full symbols) and DPPG liposomes (open symbols). Bars represent standard deviations of three independent experiments.

\section{Turbidity measurements}

The addition of berberine induced no changes in turbidity of either DPPC and DPPG liposome suspensions (data not shown). It was therefore concluded that this alkaloid caused no liposome aggregation in any of the studied model systems.

\section{DISCUSSION}

Berberine is a quaternary ammonium salt and therefore it bears a permanent positive charge in all $\mathrm{pH}$ values. The importance of electrostatic interactions in formation of complexes of berberine with polyriboadenylic acid (Yadav et al., 2005), bovine serum albumin ( $\mathrm{Hu}$ et al., 2010) and penicillin derivatives (Huang et al., 2005) has been stressed previously. The aim of the present study was to investigate the interaction of this alkaloid with neutral and negatively charged phospholipid bilayers.

As shown by DSC experiments berberine exerted almost no effect on thermotropic behavior of model membranes composed of zwitterionic DMPC if alkaloid:lipid molar ratios were up to 0.12 . Under such condition berberine did not modify lipid pretransition, the temperature of main phospholipid phase transition and half-height width of main transition peak were also unchanged.
Only the transition enthalpy was reduced in the presence of berberine. The enthalpy of main phase transition was further reduced at berberine:DMPC molar ratio 0.24 , at this concentration of the additive the pretransition disappeared, too. Similar influence on DPPC phase transitions was also reported for morphine, the most abundant alkaloid in opium (Budai et al., 2003) and also for peptides of various length containing hydrophobic leucine core and hydrophilic amino acid residues at each end (Morrow et al., 1985). It was concluded that berberine affected zwitterionic DMPC bilayer only very weakly and that high amounts of the alkaloid were required to modify thermotropic behavior of zwitterionic model membrane.

On the other hand, the significant influence of berberine on negatively charged DMPG bilayers was noticeable even at the lowest alkaloid:lipid molar ratio studied (0.02). Apart from the disappearance of the pretransition, broadening of the calorimetric peak was observed as well as the reduction of main phase transition temperature. Starting from 0.06 alkaloid:lipid molar ratio berberine induced a complex double-peak transition and a new peak was additionally registered in temperature higher than the main peak. The influence of berberine on transition enthalpy was insignificant in alkaloid:DMPG molar ratios below 0.08. The 
appearance of a new peak in the thermogram, in temperature higher than the original peak has been previously attributed to the compounds that interacted with lipid headgroup region of the bilayer probably via electrostatic interactions (Jain \& Wu, 1977). Similar modification of DMPG thermograms as seen for berberine was previously reported for cell penetrating peptide containing three tryptophans and six arginines in its sequence (Walrant et al., 2011). The authors concluded that the main mechanism of peptidemembrane interaction was electrostatic recognition between the positively charged arginines of the peptide and DMPG headgroups bearing the negative charge. It can be also concluded that electrostatic interactions played the principal role in the interaction of berberine and DMPG bilayers.

Although berberine possess very low intrinsic fluorescence in water solutions it is known to be an efficient quencher of fluorescence of other substances, such as protein tryptophans ( $\mathrm{Hu}$ et al., 2010) and thioglycolic acid-capped CdTe quantum dots (Cao et al., 2010). In the present work quenching of fluorescence of two probes, Laurdan and Prodan, by berberine was investigated. It was found that berberine quenched fluorescence of Prodan to a higher extent than Laurdan in both zwitterionic and negatively charged model membranes. It suggested that that the regions close to the surface of the bilayer were more affected by the presence of the alkaloid than the regions near phospholipid glycerol backbones where Laurdan is localized. Additionally, the upward curvature of SternVolmer plots of quenching of Prodan was noticed. This suggested that berberine molecules were present in the vicinity of the probe, quenching its fluorescence both by dynamic (collisional) and static mechanism (e.g. stacking interactions between berberine and the probe might occur). This effect was not observed for Laurdan most likely due to the lesser accessibility of this probe for berberine molecules that were present only in low amount in the membrane region where Laurdan fluorophore resided. The results of fluorescence spectroscopy experiments also corroborated the importance of electrostatic interaction for berberinemembrane interactions noticed previously by means of DSC. Quenching of both Prodan and Laurdan fluorescence by the studied alkaloid was much stronger in DPPG liposomes than in DPPC ones. It points to the fact that negatively charged phospholipid bilayers were affected by berberine to a greater extent than zwitterionic model membranes.

Turbidimetric experiments revealed that berberine did not induce liposome aggregation composed of any of the studied lipids. The alkaloid bearing a single positive charge per molecule was not able to cause DPPG liposomes to aggregate in contrast to cell penetrating peptides possessing multiple positive charges that caused aggregation in DMPG model systems (Walrant et al., 2011).

In conclusion, both DSC and fluorescence spectroscopy experiments indicated, that berberine exerted more pronounced effect in model membranes formed from negatively charged (PG), than zwitterionic lipid (PC). The electrostatic interaction between positively charged nitrogen of berberine and phosphatidylglycerol headgroup ruled the binding of the studied alkaloid to negatively charged bilayers, whereas berberine interaction with zwitterionic phosphatidylcholine membranes was weak. The results of DSC experiments together with dissimilar quenching of fluorescence of probes localized in distinct membrane regions suggested that berberine was most likely to interact with bilayer regions close to its surface. Further studies, employing lipids of various headgroups are needed to fully elucidate the interaction of berberine with phospholipid membranes.

\section{ACKNOWLEDGMENTS}

This work was financed by Polish Ministry of Science and Higher Education funds for Wrocław Medical University.

\section{REFERENCES}

Bagatolli L.A., Parasassi T., Fidelio G.D. \& Gratton E.(1999). A model for the interaction of 6-lauroyl-2-(N,Ndimethylamino)naphthalene with lipid environments: implications for spectral properties. Photochem. Photobiol., 70, 557-564.

Budai M., Szabo Z., Szogyi M. \& Grof P.(2003). Molecular interactions between DPPC and morphine derivatives: a DSC and EPR study. Int. J. Pharm., 250, 239-250.

Cao M., Liu M., Cao C., Xia Y., Bao L., Jin Y., Yang S. \& Zhu C.(2010). A simple fluorescence quenching method for berberine determination using water-soluble CdTe quantum dots as probes. Spectrochim. Acta A, 75, 1043-1046.

Freile M.L., Giannini F., Pucci G., Sturniolo A., Rodero L., Pucci O., Balzareti V. \& Enriz R.D.(2003). Antimicrobial activity of aqueous extracts and of berberine isolated from Berberis heterophylla. Fitoterapia, 74, 702-705.

Giri P. \& Kumar G.S.(2010). Isoquinoline alkaloids and their binding with polyadenylic acid: potential basis of therapeutic action. Mini Rev. Med. Chem., 10, 568-577.

Gupta S.C., Kim J.H., Prasad S. \& Aggarwal B.B. (2010). Regulation of survival, proliferation, invasion, angiogenesis, and metastasis of tumor cells through modulation of inflammatory pathways by nutraceuticals. Cancer Metastasis Rev., 29, 405-434.

Howes M-J.R. \& Houghton P.J.(2003). Plants used in Chinese and Indian traditional medicine for improvement of memory and cognitive function. Pharmacol. Biochem. Behav., 75, 513-527. 
Hu Y.J., Ou-Yang Y., Dai C.M., Liu Y. \& Xiao X.H.(2010). Binding of berberine to bovine serum albumin: spectroscopic approach. Mol. Biol. Rep., 37, 3827-3832.

Huang C.Z., Feng P., Li Y.F., Tan K.J. \& Wang H.Y.(2005). Adsorption of penicillin-berberine ion associates at a water/tetrachloromethane interface and determination of penicillin based on total internal-reflected resonance light scattering measurements. Anal. Chim. Acta, 538, 337-343.

Imanshahidi M. \& Hosseinzadeh H.(2008). Pharmacological and Therapeutic Effects of Berberis vulgaris and its Active Constituent, Berberine. Phytother. Res., 22, 999-1012.

Ivanovska N. \& Philipov S.(1996). Study on the antiinflammatory action of Berberis vulgaris root extract, alkaloid fractions and pure alkaloid. Int. J. Immunopharm., 8, 553-561.

Iwazaki R.S., Endo E.H., Ueda-Nakamura T., Nakamura C.V., Garcia L.B. \& Filho B.P.(2010). In vitro antifungal activity of the berberine and its synergism with fluconazole. Antonie Van Leeuwenhoek, 97, 201-205.

Jain M.K. \& Wu N.M.(1977). Effect of small molecules on the dipalmitoyl lecithin liposomal bilayer: III. Phase transition in lipid bilayer, J. Membr. Biol. 34, 157-201.

Jantova S., Cipak L. \& Letasiova S.(2007). Berberine induces apoptosis through a mitochondrial/caspase pathway in human promonocytic U937 cells. Toxicol. In Vitro, 21, 2531.

Krasnowska E.K., Gratton E. \& Parasassi T.(1998). Prodan as a Membrane Surface Fluorescence Probe: Partitioning between Water and Phospholipid Phases. Biophys. J., 74, 1984-1993.

Kulkarni S.K. \& Dhir A.(2010). Berberine: a plant alkaloid with therapeutic potential for central nervous system disorders. Phytother. Res., 24, 317-324.

Kuo C., Chi C. \& Liu T.(2004). The anti-inflammatory potential of berberine in vitro and in vivo. Cancer Lett., 203, 127-137.
Liu Z., Liu Q., Xu B., Wu J., Guo C., Zhu F., Yang Q., Gao G., Gong Y. \& Shao C.(2009). Berberine induces p53dependent cell cycle arrest and apoptosis of human osteosarcoma cells by inflicting DNA damage. Mutat. Res., 662, 75-83.

Morrow M.R., Huschilt J.C. \& Davis J.H.(1985). Simultaneous modeling of phase and calorimetric behavior in an amphiphilic peptide/phospholipid model membrane. Biochemistry, 24, 5396-5406.

Parasassi T., De Stasio G., d'Ubaldo A. \& Gratton E.(1990). Phase fluctuation in phospholipid membranes revealed by Laurdan fluorescence, Biophys. J., 57, 1179-1186.

Rackova L., Majekova M., Kostalova D. \& Stefek M.(2004). Antiradical and antioxidant activities of alkaloids isolated from Mahonia aquifolium. Structural aspects. Bioorg. Med. Chem., 12, 4709-4715.

Scazzocchio F., Cometa M.F., Tomassini L. \& Palmery M.(2001). Antibacterial activity of Hydrastis canadensis extract and its major isolated alkaloids. Planta Med., 67, 561-564.

Walrant A., Correia I., Jiao C.Y., Lequin O., Bent E.H., Goasdoue N., Lacombe C., Chassaing G., Sagan S. \& Alves I.D.(2011). Different membrane behaviour and cellular uptake of three basic arginine-rich peptides. Biochim. Biophys. Acta, 1808, 382-393.

Wang N., Feng J., Zhu M., Tsang C-M., Man K., Tong Y. \& Tsao S-W.(2010). Berberine induces autophagic cell death and mitochondrial apoptosis in liver cancer cells: The cellular mechanism. J. Cell. Biochem., 111, 1426-1436.

Yadav R.C., Kumar G.S., Bhadra K., Giri P., Sinha R., Pal S. \& Maiti M.(2005). Berberine, a strong polyriboadenylic acid binding plant alkaloid: spectroscopic, viscometric, and thermodynamic study. Bioorg. Med. Chem., 13,165-174. 